\title{
PHOTOELECTRON DIFFRACTION AND SYNCHROTRON RADIATION
}

\author{
J. OSTERWALDER \\ Institut de Physique, Université de Fribourg, 1700 Fribourg, Switzerland
}

The basic concepts of photoelectron diffraction are briefly introduced. The increasing number of synchrotron beam lines devoted to this type of experiments is explained by the variety of surface phenomena that can be studied. New developments, such as the measurement of full hemispherical angular distributions, photoelectron holography, energy-scanned photoelectron diffraction, chemical-state resolved photoelectron diffraction, and spin-polarized photoelectron diffraction all benefit from the special attributes of synchrotron radiation.

PACS numbers: $61.14 .-\mathrm{x}, 79.60 .-\mathrm{i}$

\section{Introduction}

Core level photoelectrons emitted from single crystals show very strong anisotropies due to the phenomenon of photoelectron diffraction $[1,2]$. The emitting atom represents an internal, localized source of electrons, and forward scattering and wave interference produce angular patterns that contain information on the short-range crystalline structure. If the photoelectron energy is a few hundred $\mathrm{eV}$ or more, bond directions within the surface region can be readily identified by the appearance of distinct maxima in the angular distribution $[1,2]$. This is due to a quite general property of the atomic form factor, i.e. the strong scattering into the forward direction by the attractive ion potentials of atoms near the emitter, which has also been termed "forward focusing" [3]. In addition, interference effects due to path length differences of source and scattered waves, which can provide bond length information, occur at non-zero scattering angles. They are dominant at energies below $200 \mathrm{eV}$, where the atomic form factors are less anisotropic. Both these effects are illustrated in Fig. 1.

While a great number of experiments can be done with laboratory sources, synchrotron radiation offers some specific advantages that will be emphasized in this paper. First, it has been recognized in the past few years that the measurement of data sets over a large part of the solid angle above the surface, i.e. of two-dimensional intensity maps, strongly increases the power of this surface structural technique [4-7]. A high photon flux is therefore needed in order to keep 
a)
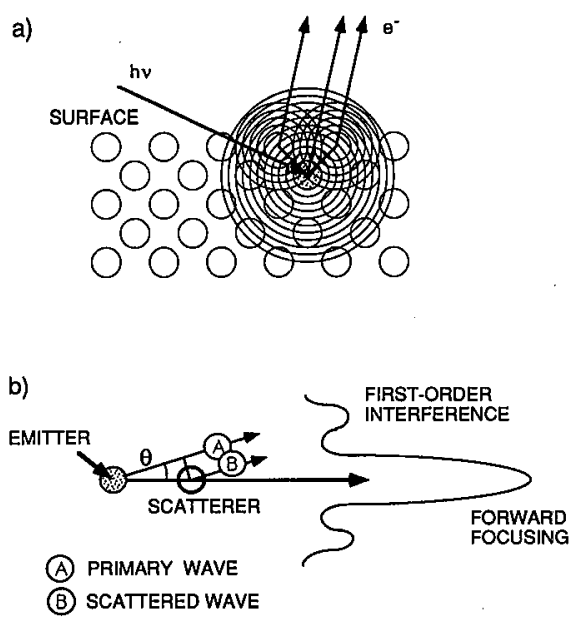

Fig. 1. (a) Illustration of photoelectron wave interference upon photoexcitation within the surface. (b) Important scattering processes contributing to photoelectron diffraction: forward focusing and first-order interference. In the latter case, the phases of primary and scattered waves differ by $2 \pi$.

measuring times within reasonable bounds. Second, the tunability of the photon energy permits to measure angular distributions at various photoelectron energies, and therefore to separate the interference effects, which are energy-dependent, from forward-focusing effects. It has very recently been claimed that atomic positions in the vicinity of the photoemitter can directly be obtained from this interference structure by two-dimensional Fourier transformation techniques $[8,9]$. This new concept has been termed "photoelectron holography", in analogy to its somewhat related counterpart in optics. Energy-scanned photoelectron diffraction experiments that monitor the intensity at a fixed emission angle are also briefly discussed, as well as spin-polarized photoelectron diffraction. Third, high-energy resolution of better than $0.5 \mathrm{eV}$ opens the possibility of chemical-state resolved photoelectron diffraction. Atoms of the same kind but having different chemical or structural surroundings appear with shifted positions in the spectra, typically 0.1 to $5 \mathrm{eV}$ apart [10]. If the energy resolution is sufficient to separate these contributions, the structural order around these species can be determined independently.

\section{Experimental aspects}

Photoelectron diffraction experiments at synchrotron beam lines can in principle be performed in the same geometry as used in a typical laboratory setup. In order to monitor the photoelectron flux as a function of emission direction relative to the sample crystal axes, some angle-resolving energy analyzer - usually a hemispherical-sector analyzer [11] - is either mounted at a fixed position relative to the exciting photon source while the crystal is rotated, or alternatively a smaller analyzer is moved above a fixed crystal. The first solution has usually the 
advantage of higher sensitivity, while the second permits also to exploit the dependence of diffraction effects on the photon polarization, particularly if the crystal can still be rotated [12]. Evidently, data acquisition times in these scanning-type experiments can be rather long, depending on the intensity of the measured signal. The high photon flux of synchrotron radiation is thus advantageous.

Recently, more complex analyzer systems have been designed, displaying either a full polar [13] or azimuthal [14] section or a large two-dimensional solid angle range [15] of the photoelectron angular distribution. Here, the high brilliance of synchrotron radiation, and associated with it the small illuminated spot size, are essential for proper performance of the electron optical system, and data acquisition times are greatly reduced. On the other: hand, some of these designs suffer from very low signal-to-background ratios in the higher-energy regime and from energy-dependent angular distortions [15].

\section{Theoretical approaches}

In order to draw quantitative structural conclusions, it is the usual procedure to model the measured angular distributions by some sort of scattering calculations with a suitable surface atomic structure. The calculations are repeated until the best fit is achieved, thus optimizing the structural parameters.

There is an ongoing debate whether multiple scattering (MS) of the outgoing photoelectron needs to be considered absolutely, or whether the single-scattering events dominate the diffraction patterns sufficiently to permit structural analysis with a single-scattering cluster (SSC) formalism [1]. Most work up to now has been done along this latter line, and some particular cases where MS shows up strongly in the data have been isolated [16-19].

In the SSC theory first derived by Lee [20], a coherent summation is carried out of the primary, unscattered wave $\phi_{0}$ with all the wavelets $\phi_{j}$ scattered at atomic positions $r_{j}$. The photocurrent $I(k)$ along the emission direction $k$ is then obtained as the modulus of the complex sum. In the simplest version, which involves emission from an isotropic $s$ state and scattering of plane waves (PW) [1], one uses

$$
\begin{aligned}
I(k) & =\left|(\widehat{\varepsilon} \cdot \widehat{k}) \mathrm{e}^{-L_{0} / 2 \Lambda_{\mathrm{e}}}+\sum_{j}\left(\widehat{\varepsilon} \cdot \widehat{r}_{j}\right) \mathrm{e}^{-L_{j} / 2 \Lambda_{\mathrm{c}}} W_{j} \frac{f_{j}\left(\theta_{j}\right)}{r_{j}} \mathrm{e}^{\mathrm{i} k r_{j}\left(1-\cos \theta_{j}\right)}\right|^{2} \\
& + \text { TDS. }
\end{aligned}
$$

Here, $(\widehat{\varepsilon} \cdot \widehat{k})$ and $\left(\widehat{\varepsilon} \cdot \widehat{r}_{j}\right)$ correspond to the angular part of the photoemission matrix element associated with the photon polarization unit vector $\widehat{\varepsilon}$, giving thus the relative excitation probability along the observation direction and into the direction of scatterer $r_{j}$. The terms $\mathrm{e}^{-L_{j} / 2 \Lambda_{\mathrm{e}}}$ and $W_{j}$ describe the inelastic attenuation of photoelectron flux along the path length $L_{j}$ (inelastic mean free path $\Lambda_{\mathrm{e}}$ ) and thermal effects (Debye-Waller factor), respectively. The spherical character of the primary wave is contained in the $1 / r_{j}$ factor, while the scattering potential of each atom is represented by a plane-wave complex form factor $f_{j}\left(\theta_{j}\right)$, including a scattering phase shift [21]. The complex exponential is the interference term, and 
TDS - the thermal diffuse scattering term that balances products of $W_{j}$ terms of the same site $\boldsymbol{r}_{j}$ appearing when calculating the modulus explicitly [1].

Efficient computer programs were developed to include further instrumental angular broadening, polarized or unpolarized radiation, and spherical-wave scattering of proper angular momentum final states for $s, p, d$ or $f$ emission [22].

\section{Two-dimensional intensity measurements}

In order to illustrate the character of complete angular distribution patterns of photoelectrons (and Auger electrons) at different energies, Fig. 2 gives four experimental patterns, excited with laboratory X-ray sources, from $\mathrm{Cu}(001)$ [23]. The intensity maps are plotted in the stereographic projection, with maximum

a) Cu $28(\mathrm{Mg} \mathrm{K} \alpha) 156.5 \mathrm{oV}$

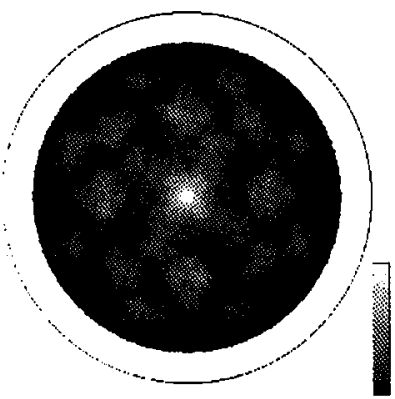

b) $\mathrm{Cu} 2 \mathrm{p}_{32}(\mathrm{Mg} \mathrm{K \alpha}) 321.3 \mathrm{eV}$

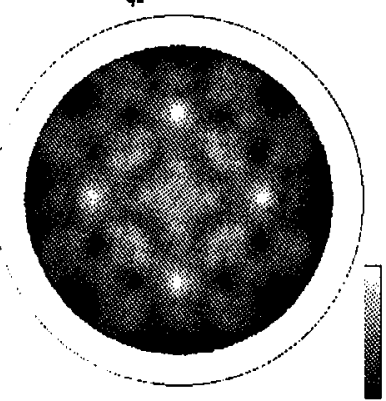

c) Cu L, V V (Mg Ka) $918.6 \mathrm{eV}$

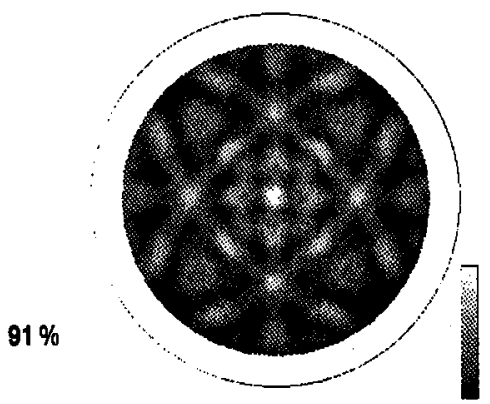

$64 \%$

d) Cu 3p (Si Ka) $1664.2 \mathrm{eV}$

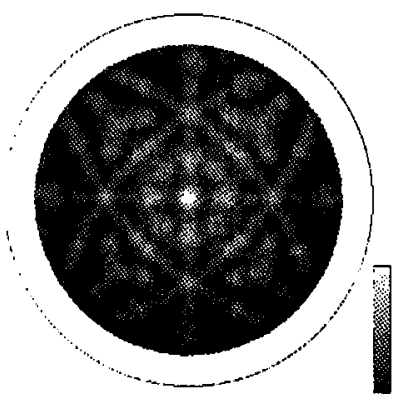

$6 \%$

Fig. 2. Full hemispherical photoelectron angular distributions from $\mathrm{Cu}(001)$, excited by $\mathrm{Mg} K_{\alpha}(1253.6 \mathrm{eV})$ and $\mathrm{Si} K_{\alpha}(1740 \mathrm{eV})$ laboratory X-ray sources, and obtained by crystal rotation. Four different transitions at different kinetic energies are shown. The plots are given in the stereographic projection, in a linear grey scale with maximum intensities in white.

and minimum intensities represented by white and black, respectively. At high energies (Figs. 2c and 2d), the strong forward peaking of the scattering essentially leads to a projection of the crystal structure from a lattice site near the surface onto the hemisphere above the surface. This becomes evident by comparing the 
$\mathrm{Cu} 3 p$ distribution at $1664 \mathrm{eV}$ to such a projection of a face-centered cubic lattice shown in Fig. 3. Most low-index directions are present in the data as local intensity

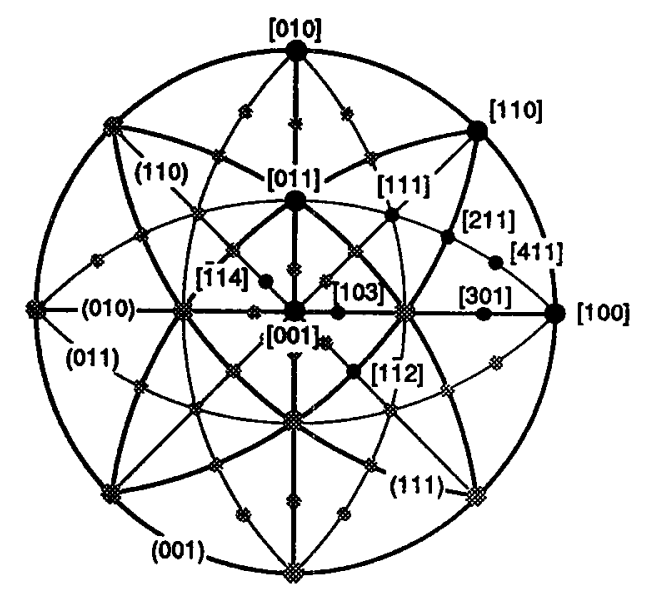

Fig. 3. The projections of some high-density crystal planes (lines) and the major low-index directions (spots) of a face-centered cubic (001) surface are given in the same format as the data in Fig. 2.

maxima, and the projections of high atomic density crystal planes appear as bright, narrow bands. This simple interpretation was found valid on many other crystal surfaces [4-7], and can also be used to analyze similar data from ultrathin films [24].

As the energy of the photoelectrons decreases and forward scattering becomes less important, the projected real-space image gets more and more diffuse. The forward-focusing maxima become broader, and so do the intensity bands which at the same time become more and more modulated by interference effects. In the $\mathrm{Cu} 2 s$ pattern at $156 \mathrm{eV}$, the correspondence to the crystal lattice is almost completely lost, except for the strong maximum along the [001] direction in the center. More careful inspection of this intensity map - note that the cross-like structure dominating the center of the plot is significantly rotated counterclockwise away from the (110) mirror plane - shows that even the two mirror plane symmetries of this (001) surface are broken, an effect that can be well explained by photon polarization effects in the excitation matrix element $[23,25]$. Depending on the particular transition involved, the excited electron wave - also termed source wave - will have different angular momenta prior to scattering, corresponding to different degrees of anisotropy. The spherical harmonics, associated with these angular momenta and with a particular polarization axis therefore introduce a weighting of the various scattering events that occur on propagating to the surface. The patterns are thus mainly the result of a complex interplay between interference effects and source effects.

The use of synchrotron radiation of tunable energy permits to measure different transitions, i.e. different final state angular momenta, at constant energy, and 
should therefore be very important to further our understanding of this interplay. One such study has recently been published by Terminello and Barton [26], comparing the patterns of $\mathrm{Cu} M_{2,3} M_{4,5} M_{4,5}$ and $\mathrm{Cu} 3 p$ emission from $\mathrm{Cu}(001)$, both at $60 \mathrm{eV}$. Their results indicate that the source wave effects are very important at this energy.

\section{Photoelectron holography}

It was recently pointed out by Szöke that there are conceptual similarities between optical holography and photoelectron diffraction [8]. The primary, unscattered photoelectron wave and the sum of all the scattered wavelets take the roles of the reference and object waves, respectively. Barton further showed [9], using the Helmholtz-Kirchhoff theorem, that the atomic positions of atoms around a photoemitter can in principle be found by a phased Fourier transform of the hemispherical photoelectron distribution. This was confirmed by reconstructing real-space images from photoelectron and Auger electron patterns measured from clean substrates, e.g. $\mathrm{Cu}(001)$ [27] and $\mathrm{Si}(111)$ [28], at rather high energies of the order of $1 \mathrm{keV}$. The images so obtained indeed show structures near known atomic positions. However, considerable structure is also observed at non-physical sites, and various procedures for improving image resolution and fidelity were introduced [29-31].

The bond length information is contained in the interference structure. Since we know that at $1 \mathrm{keV}$ the diffraction patterns are highly dominated by forward focusing, one may wonder how such a Fourier technique can still extract the proper atomic positions. Stuck et al. [32] applied a related inversion technique to emission patterns measured from $\mathrm{Cu}(001)$ at seven different energies ranging from $768 \mathrm{eV}$ to $1740 \mathrm{eV}$ (taken from the same data set as those of Fig. 2). In Fig. 4 the main results of their study are given. Radial distribution curves of the reconstructed real-space image are shown along the [011] nearest-neighbour direction, both as a function of distance in $\AA$ (right) and of the number of wavelengths $(r / \lambda$, left). The scattered-wave-included Fourier transform (SWIFT) method [30] was used in order to suppress the disturbing effects of the strong anisotropy in the complex scattering factor, which is known to affect both peak position and shape [29-31]. The right panel of Fig. 4 shows that there is a peak in image intensity near the true atomic position (dashed line) for the lower three curves, including the Auger transition at $918 \mathrm{eV}$ used in previous work [27]. However, considering all curves makes this imaging of the atom position appear fortuitous. More significantly, the left panel demonstrates that all major features do not move on the wavelength scale. This indicates that they are due to the symmetry of the pattern, which is determined by the forward-projected crystal structure, rather than due to energy-dependent interference structure. In this energy range, photoelectron holography using substrate signals needs thus to be considered very critically.

New suggestions employing measured intensity maps at several energies, which are then weighted by energy-dependent phase factors and subject to a combined Fourier transform [33,34], appear still promising, particularly at lower energies where interference effects are stronger. It should be pointed out here that 

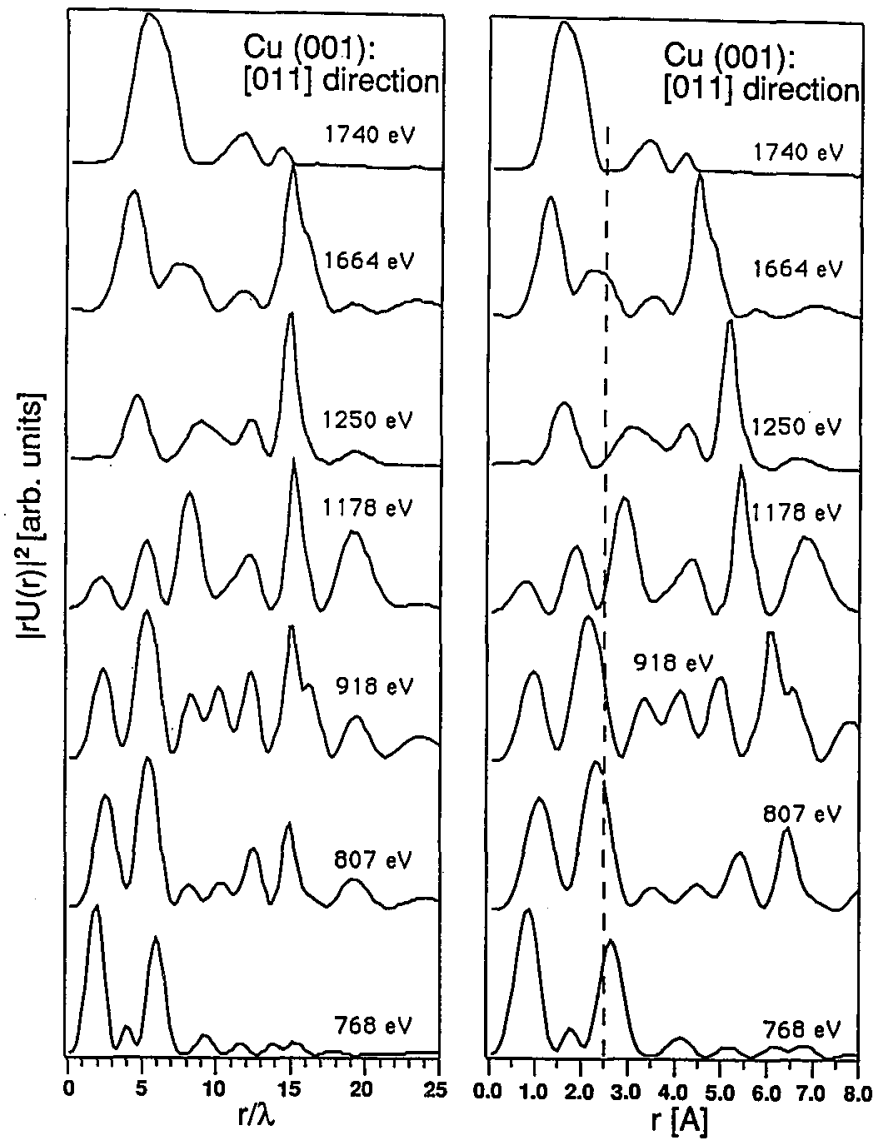

Fig. 4. The real-space radial distribution curves obtained by holographic inversion (using SWIFT corrections) of photoelectron diffraction data from $\mathrm{Cu}(001)$ at seven different energies are shown' along the nearest-neighbour [011] direction. The radial probability distribution is plotted against (a) the distance $r$ in units of the electron wavelength $\lambda$, and (b) the distance measured in $\AA$. The correct nearest-neighbour distance is indicated by the dashed line.

Fourier methods were successfully applied to the analysis of energy-scanned photoelectron diffraction data [35] (see below), where the intensity modulations are created by the energy-dependent interference effects along a fixed direction. The new approach represents thus an extension of this technique to include all emission angles. Obviously, the data sets needed for this analysis scheme are very large and will require the use of synchrotron radiation.

Alternatively, the single-energy holographic approach to photoelectron diffraction may be useful if applied to emission from adsorbate atoms [9], in which case forward-focusing peaks are often missing due to the two-dimensional character of these structures. The patterns, although very weakly anisotropic, are here entirely composed of interference structure. 


\section{Energy-scanned photoelectron diffraction}

When adsorbate structures are to be solved, there is often a small number of possible adsorption sites to consider, and the main problem is to discriminate among these, and to measure the bond distance from the substrate. This can be done with high precision using backscattering interference in energy-scanned photoelectron diffraction of adsorbate emission [36, 37]. One chooses the electron emission direction along a likely backscattering direction, i.e. a direction that involves scattering off near-neighbour atoms by $180^{\circ}$. In the energy range between 50 and $400 \mathrm{eV}$ typically used, the backscattering amplitude is sufficiently strong to produce oscillations that are dominated by the terms

$$
I(|k|)=\left|1+\mathrm{e}^{-r_{j} / A_{\mathrm{e}}} W_{j} \frac{f_{j}\left(\pi_{j}\right)}{r_{j}} \mathrm{e}^{\mathrm{i} 2 k r_{j}}\right|^{2}+\mathrm{TDS},
$$

as can be readily seen from Eq. (1). The main frequency will thus be given by the $\cos \left(2 k r_{j}\right)$ term and the neighbour distance $r_{j}$ can be determined.

Much of the existing photoelectron diffraction work at synchrotron sources has been devoted to this type of experiment, which takes advantage of the tunability of the photon energy and which is sometimes referred to as angle-resolved photoemission fine structure (ARPEFS). In spite of the strong resemblance to extended X-ray absorption fine structure (EXAFS), this is an angular resolved experiment permitting a three-dimensional refinement of the structure.

Dippel et al. [38] have very recently shown that a combination of angleand energy-scanned photoclectron diffraction experiments can be very efficiently used for determining adsorbate structure. They exploit the strong oscillations of the backscattering intensity with energy to locate the adsorbate bond direction. Specifically, they take some reasonable assumption for the bond length and calculate the backscattering intensity oscillations. In the next step they perform angular scans at two photoelectron kinetic energies corresponding to an adjacent maximum and minimum in the backscattering curve. The ratio curve of the two angular scans shows a distinct maximum along the adsorbate bond direction corresponding to the chosen bond length. The structure of adsorbates can thus be determined in a similarly transparent way as using forward focusing for subsurface emitters.

\section{Chemical-state resolved photoelectron diffraction}

Photoemitters bonded to different atoms, or having different coordination numbers, appear at differing energy positions in a spectrum [10]. If the spectrometer resolution is sufficient to separate these components, the angular distribution of each can be measured separately. This should in principle permit to determine the short-range structure around each type of emitter present in a surface. A special case of particular interest is the possibility of measuring surface core-level shifts, in which case electrons from surface atoms appear with different binding energies. These surface signals constitute a very sensitive probe for studying surface reconstruction [39].

As a nice example we give here a very recent study of the $(2 \times 8)$ reconstructed surface of $\mathrm{Ge}(111)$ using synchrotron radiation by Patthey et al. [40]. The Ge $3 d$ 
line, excited at $100 \mathrm{eV}$ photon energy, is found to exhibit variations in line shape with emission angle. As is shown in Figs. $5 \mathrm{a}$ and $5 \mathrm{~b}$, this can be accounted for by superimposing two surface signals $S 1$ and $S 2$ with the bulk signal, shifted by $0.27 \mathrm{eV}$ and $0.73 \mathrm{eV}$ to lower binding energy, respectively. All of them show a
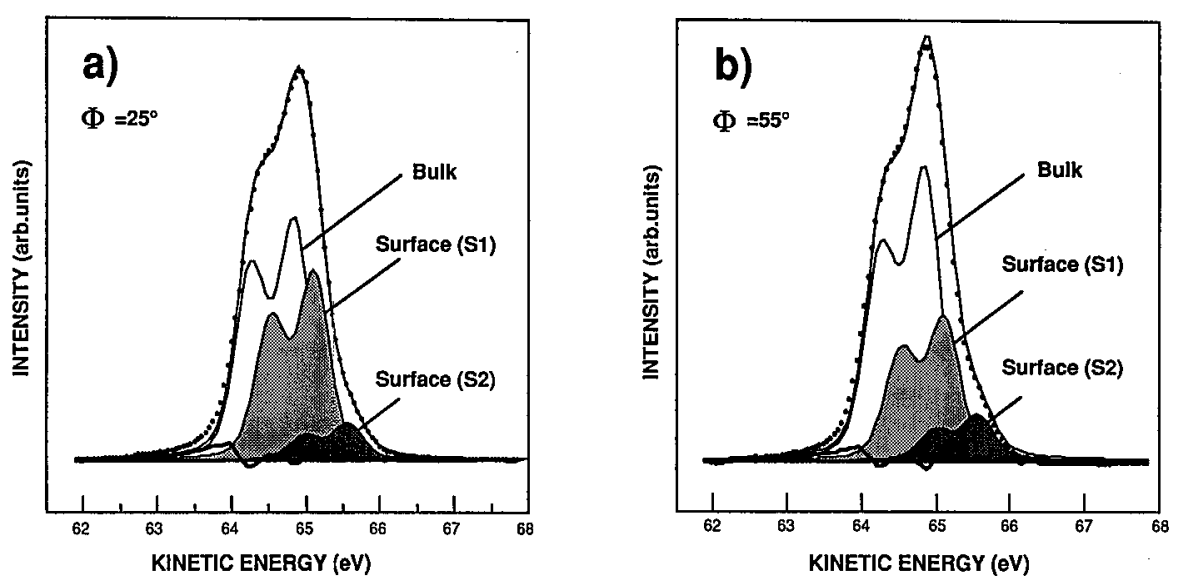

Fig. 5. $\mathrm{Ge}(111)-\mathrm{c}(2 \times 8) 3 d$ core-level spectra measured at $100 \mathrm{eV}$ photon energy (a) at an azimuthal angle $\phi=25^{\circ}$ (see Fig. 6) and (b) at $\phi=55^{\circ}$, showing the results of the deconvolution into a bulk and two surface components.

constant spin-orbit splitting. The spectra were taken at a polar emission angle $\theta$ of $51^{\circ}$ off normal and at two different azimuths, $[-110]$ and $30^{\circ}$ off. The structure of this reconstructed surface was solved by surface X-ray diffraction [41], but there was some controversy about the assignment of the two surface-shifted components [42, 43]. At the top of Fig. 6, side views of the local surface geometry are given according to the model of van Silfhout et al. [41], indicating that the local bonding configuration involves adatoms on a double layer and restatoms on a single layer. Figure 6a gives the assignment of the $S 1$ component to adatoms and 1st-layer atoms, and $S 2$ to restatoms. Alternatively, $S 1$ has been associated with restatoms and 1st-layer atoms and $S 2$ with adatoms (Fig. $6 \mathrm{~b}$ ).

Patthey et al. [40] demonstrate that the spectral components reveal their origin by their unique diffraction patterns associated with their local geometry. In the bottom part of Fig. 6 , the intensity variations with azimuthal angle $\left(\theta=51^{\circ}\right)$ of the total $3 d$ signal and of the three curve-fitted components (bulk, $S 1$ and $S 2$ ) are given (full lines). All of them show significantly different patterns. The dashed curves give results of single-scattering calculations using spherical-wave scattering, all using the same atomic cluster with the surface reconstructed as indicated, but with different atoms acting as photoemitters. For the total and bulk signals fair agreement of peak positions is found, with some problems as for relative intensities commonly encountered in single-scattering calculations [1]. For the surface components, the two different assignments are tested against each 
a)

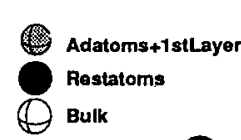

b)
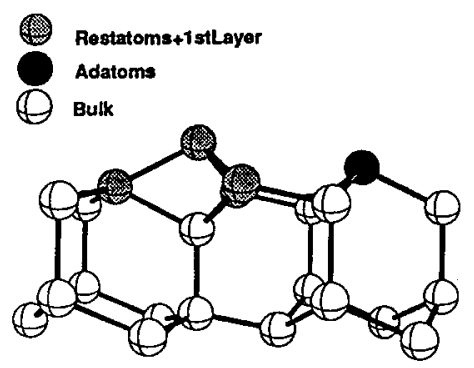
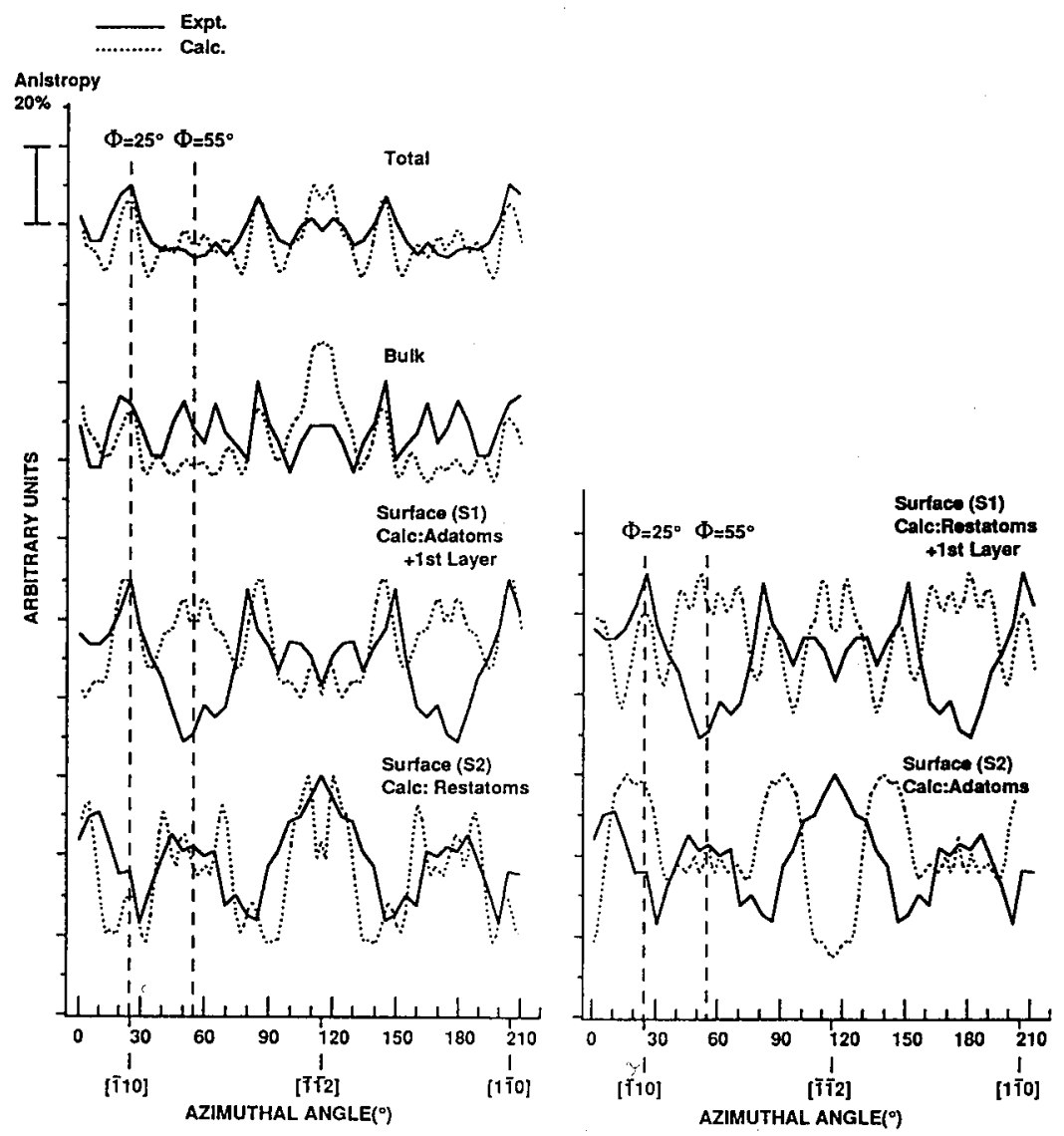

Fig. 6. (a) Side view of the local geometry within the $\mathrm{c}(2 \times 8)$ reconstruction of $\mathrm{Ge}(111)$. Below there are the azimuthal photoelectron diffraction curves (solid lines) for the total, bulk, surface $S 1$ and surface $S 2$ peaks (see Fig. 5). On these there are superimposed (dashed lines) the results of the calculations assuming the structure shown. Here the calculation for peak $S 1$ includes the signal from the adatoms and 1st-layer atoms and for peak $S 2$ the signal from the rest atoms. (b) The same view of the $\mathrm{c}(2 \times 8)$ structure, but with a different assignment for the surface peaks. 
other, and the data clearly favor the one shown in Fig. 6a, with $S 2$ as emission from restatoms.

\section{Spin-polarized photoelectron diffraction}

The scattering of electrons off magnetic ions depends on the relative spin orientation, with spin asymmetries becoming rather important at energies of $100 \mathrm{eV}$ and below [44]. Sinkovic and Fadley [45] used the multiplet-split $3 s$ photoelectrons from $\mathrm{Mn}^{2+}$ ions as an internal spin-polarized electron source. Here, the exchange interaction of the high-spin $3 d^{5}$ shell and the $3 s$ hole leads to two peaks in the energy spectrum, split by $6 \mathrm{eV}$, and highly spin-polarized with respect to the $3 d$ local moment of the photoemitter. Observed differences in the angular distributions of the two peaks are thus partly due to the spin dependence in the scattering. It is easily seen that only the relative orientation of the emitter and scatterer magnetic moments is relevant. This type of measurement is thus sensitive to the local spin-spin correlation.

Rather than measuring the angular dependence of the two multiplet peaks, Sinkovic et al. [46] measured the temperature dependence of the peak ratio at fixed emission angle in $\mathrm{Mn} 3 s$ emission from antiferromagnetic $\mathrm{KMnF}_{3}(011)$. A rather abrupt change with temperature was found in this ratio which cannot be explained in terms of Debye-Waller factors (Eq. (1)), indicating thus a change in the local spin order. This transition occurs well above the Néel temperature of the crystal. A similar phenomenon was found on antiferromagnetic $\mathrm{MnO}$ [47].

Experiments of this kind are rather unique in that they appear to probe the short-range magnetic order in ferromagnets or antiferromagnets. Most experiments have so far been carried out with laboratory sources and thus suffer from very low signal-to-background ratios, low energy resolution, and limited choice of suitable photon energies, which has made them a very tedious procedure. The use of synchrotron radiation should here open the field for the application to many more magnetic systems.

\section{Conclusions}

At the present time we are aware of almost a dozen synchrotron beam lines that are currently devoted to, or being prepared to carrying out photoelectron diffraction experiments. This expresses the widespread interest in this rather young technique, which is unique because it combines chemical, structural and in special cases also magnetic sensitivity.

\section{Acknowledgements}

We would like to express our gratitude to all our coworkers at the University of Fribourg for their significant contribution to this review, and to E. Bullock for providing some figures prior to publication. Special thanks are to C.S. Fadley who introduced us into this field, to S. Hüfner for his many ideas, and to L. Schlapbach for continuous support. This work was financed by the Schweizerische Nationalfonds. 


\section{References}

[1] C.S. Fadley, in: Synchrotron Radiation Research: Advances in Surface Science, Ed. R.Z. Bachrach, Plenum, New York 1990, in press.

[2] W.F. Egelhoff, Jr., Crit. Rev. Solid State Mater. Sci. 16, 213 (1990).

[3] H.C. Poon, S.Y. Tong, Phys. Rev, B 30, 6211 (1984).

[4] H. Li, B.P. Tonner, Phys. Rev. B 37, 3959 (1988).

[5] M. Seelmann-Eggebert, H.J. Richter, Phys. Rev. B 43, 9578 (1991).

[6] D.G. Frank, N. Battina, R. Golden, F. Lu, A.T. Hubbard, Science 247, 182 (1990).

[7] J. Osterwalder, T. Greber, A. Stuck, L. Schlapbach, Phys. Rev. B 44, 13764 (1991).

[8] A. Szöke, in: Short Wavelength Coherent Radiation: Generation and Applications, AIP Conf. Proc. No. 147, Ed. D.T. Attwood, J. Boker, AIP, New York 1986.

[9] J.J. Barton, Phys. Rev. Lett. 61, 1356 (1988).

[10] W.F. Egelhoff, Jr., Surf. Sci. Rep. 6, 253 (1987).

[11] C.E. Kuyatt, J.A. Simpson, Rev. Sci. Instrum, 38, 103 (1967); M.P. Seah, Surf. Interface Anal. 2, 222 (1980).

[12] B. Sinkovic, P.J. Orders, C.S. Fadley, R. Trehan, Z. Hussain, J. Lecante, Phys. Rev. $B$ 30, 1833 (1984).

[13] R.C.G. Leckey, J.D. Riley, Appl. Surf. Sci. 22/23, 196 (1985).

[14] S. Kono, Oyo Buturi 54, 913 (1985).

[15] D.E. Eastman, J.J. Donelon, N.C. Hien, F.J. Himpsel, Nuclear Instrum. Methods 172, 327 (1980); H. Daimon, Rev. Sci. Instrum. 59, 545 (1988).

[16] S.Y. Tong, H.C. Poon, D.R. Snider, Phys. Rev. B 32, 2096 (1985).

[17] M.L. Xu, J.J. Barton, M.A. Van Hove, Phys. Rev. B 39, 8275 (1989).

[18] W.F. Egelhoff, Jr., Phys. Rev. Lett. 59, 559 (1987).

[19] J. Osterwalder, T. Greber, S. Hüfner, L. Schlapbach, Phys. Rev. B 41, 12495 (1990). [20] P.A. Lee, Phys. Rev. B 13, 5261 (1976).

[21] M. Fink, A.C. Yates, At. Data 1, 385 (1970); M. Fink, J. Ingram, At. Data 4, 129 (1972); D. Gregory, M. Fink, At. Data Nucl. Data Tables 14, 39 (1974).

[22] D.J. Friedman, C.S. Fadley, J. Electron Spectrosc. Relat. Phenom. 51, 689 (1990).

[23] D. Naumovic, A. Stuck, T. Grcbcr, J. Osterwalder, L. Schlapbach, submitted to Phys. Rev. B.

[24] D. Naumovic, A. Stuck, T. Greber, J. Osterwalder, L. Schlapbach, Surf. Sci., in press, 1992.

[25] T. Greber, J. Osterwalder, S. Hüfner, L. Schlapbach, Phys. Rev. B 45, 4540 (1992).

[26] L.J. Terminello, J.J. Barton, Science 251, 1218 (1991).

[27] G.R. Harp, D.K. Saldin, B.P. Tonner, Phys. Rev. B 42, 9199 (1990).

[28] G.S. Herman, S. Thevuthasan, T.T. Tran, Y.J. Kim, C.S. Fadley, Phys. Rev. Lett. 68, 650 (1992).

[29] S. Thevuthasan, G.S. Herman, A.P. Kaduwela, R.S. Saiki, Y.J. Kim, W. Niemczura, M. Burger, C.S. Fadley, Phys. Rev. Lett. 67, 469 (1991).

[30] B.P. Tonner, Zhi-Lan Han, G.R. Harp, D.K. Saldin, Phys. Rev. B 43, 14423 (1991).

[31] H. Huang, Hua Li, S.Y. Tong, Phys. Rev. B 44, 3240 (1991). 
[32] A. Stuck, D. Naumovic, H.A. Aebischer, T. Greber, J. Osterwalder, L. Schlapbach, Surf. Sci. 264, 380 (1992); A. Stuck, D. Naumovic, T. Greber, J. Osterwalder, L. Schlapbach, Surf. Sci., in press, 1992.

[33] J.J. Barton, Phys. Rev. Lett. 67, 3106 (1991).

[34] S.Y. Tong, Hua Li, H. Huang, Phys. Rev. Lett. 67, 3102 (1991).

[35] J.J. Barton, C.C. Bahr, Z. Hussain, S.W. Robey, J.G. Tobin, L.E. Klebanoff, D.A. Shirley, Phys. Rev. Lett. 51, 272 (1983).

[36] M. Sagurton, E.L. Bullock, C.S. Fadley, Surf. Sci. 182, 287 (1987).

[37] D.P. Woodruft, in: Angle Resolved Photoemission, Ed. S.D. Kevan, Elsevier, Amsterdam 1992.

[38] R. Dippel, D.P. Woodruff, X.-M. Hu, M.C. Asensio, A.W. Robinson, K.-M. Schindler, K.-U. Weiss, P. Gardner, A.M. Bradshaw, Phys. Rev. Lett. 68, 1543 (1992).

[39] D. Sebilleau, M.C. Desjonqueres, D. Chavau, C. Guillot, J. Lecante, G. Treglia, D. Spanjaard, Surf. Sci. 185, L527 (1987).

[40] L. Patthey, E.L. Bullock, K. Hricovini, Surf. Sci., in press, 1992.

[41] R.G. van Silfhout, J.F. van der Veen, C. Norris, J.E. MacDonald, Faraday Discuss. Chem. Soc. 89, 169 (1990).

[42] J. Aarts, A.-J. Hoeven, P.K. Larsen, Phys. Rev. B 38, 3925 (1988).

[43] R.D. Schnell, F.J. Himpsel, A. Bogen, D. Rieger, W. Steinmann, Phys. Rev. B 32, 8052 (1985).

[44] J.A.D. Mathew, Phys. Rev. B 25, 3326 (1982).

[45] B. Sinkovic, C.S. Fadley, Phys. Rev. B 31, 4665 (1985).

[46] B. Sinkovic, B. Hermsmeier, C.S. Fadley, Phys. Rev. Lett. 55, 1227 (1985).

[47] B. Hermsmeier, J. Osterwalder, D.J. Friedman, C.S. Fadley, Phys. Rev. Lett. 62, 478 (1989). 